\title{
Association of Second Hand Smoking with Mental Disorders in Children- An Egyptian Study
}

Eman A Zaky ${ }^{1 *}$, Eman M Fouda ${ }^{1}$, Enas S Nabih², Omneya I Youssef ${ }^{1}$ and Nermin H Mohamed ${ }^{1}$

${ }^{1}$ Department of Pediatrics, Faculty of Medicine, Ain Shams University, Cairo, Egypt

${ }^{2}$ Department of Medical Biochemistry \& Molecular Biology, Faculty of Medicine, Ain Shams University, Cairo, Egypt

\begin{abstract}
Background: Second hand smoke (SHS) might affect mental health of children as many mental disorders have an onset in youth, a time when SHS exposure is high. Objectives: Investigation of the potential association of SHS exposure with children's mental health disorders.

Methodology: Forty five SHS exposed Egyptian children were enrolled as group I; all of them had at least one smoking parent and or were exposed to environmental tobacco smoke while group II included thirty age and sex well matched controls, with neither history of parental smoking nor exposures to environmental tobacco smoke. For all studied children, complete history taking, thorough clinical examination, psychometric assessment using Pediatric Symptom Checklist (PSC), Strengths and Difficulties Questionnaire (SDQ), and IQ measurement were done. DSM IV TR criteria were used to confirm any suspected behavioral and or psychiatric disorder. Laboratory assessment of second hand smoke was carried out measuring urinary cotinine levels.

Results: SHS exposed children had significantly higher mean value of urinary cotinine level, total PSC, and SDQ scores compared to controls ( $p<0.001, p<0.001, p<0.05$ respectively). Mean values of Attention Deficit Hyperactivity Disorder (ADHD), internalization, and externalization behaviors' sub-scores of PSC were significantly higher in SHS children compared to controls. Meanwhile, mean values of emotion, conduct, ADHD, and peer problem SDQ subscores were higher in SHS children compared to controls but the comparison was only statistically significant for ADHD sub-score while prosocial problem sub-score was significantly lower in SHS children compared to controls. In studied SHS children, degree of smoking significantly positively correlated with PSC internalization behavior subscore and emotion subscore of SDQ while smoking index significantly positively correlated with ADHD subscore of SDQ. Meanwhile, prosocial subscore of SDQ and urinary cotinine level were significantly negatively correlated. In conclusion, SHS exposure significantly compromised mental health of the studied sample of Egyptian SHS exposed children.
\end{abstract}

Keywords: Secondhand Smoke; Strengths and difficulties Questionnaire; Pediatric Symptom Checklist; Childhood Mental Health

\section{Introduction}

Non-smokers inhale the mixture of side stream smoke given off by a smoldering ciga $\neg$ rette and mainstream smoke exhaled by a smoker, a mixture now referred to as "secondhand smoke" (SHS) or "environmental tobacco smoke. It contains many poisons, including nicotine (a pesticide), carbon monoxide, ammonia, formaldehyde, hydrogen cyanide, nitrogen oxides, phenol, sulfur dioxide, and others [1].

SHS exposure, either in utero or during childhood, has been causally linked to numerous adverse health outcomes, and is a leading preventable cause of both low birth weight and sudden infant death syndrome, and a major contributor to increased rates of lower respiratory infections, otitis media, reduced lung functions, and increased asthma severity and more frequent asthma attacks [2,3]. Recently, associations between SHS exposure and other childhood problems including increased rates of dental caries and the metabolic syndrome have been identified [4].

Because many mental disorders have an onset in youth at a time when SHS exposure is high, it is critical to consider how SHS may be affecting the mental health of children and adolescents, studies indicated that prenatal tobacco and childhood SHS exposure are associated with child behavioral problems, including internalizing and externalizing behaviors, ADHD, and conduct disorder [4]. It was reported that primate postnatal tobacco smoke exposure leads to changes in brain cell development similar to prenatal nicotine exposure [5].

The current study aimed at investigating the potential association between SHS exposure and emotional, behavioral, and or psychiatric problems in a group of Egyptian children as to the authors' knowledge, such an association have not been studied before in our country.

\section{Study Design and Methodology}

The current cross sectional study was carried out in accordance to the code of ethics of the World Medical Association Declaration of Helsinki [6], for experiments involving humans. Written consent of legal caregivers of enrolled children was taken and the study protocol was approved by Ain Shams Faculty of Medicine Ethical Committee. Seventy five Egyptian children were enrolled whom their caregivers agreed to their participation in the study after explaining its objective to them. They were consecutively recruited from children attended the Outpatient Clinic, Children's Hospital, Ain Shams University for growth monitoring or regular check up during the period of the clinical part of the study from February 2012 to January 2013.

\section{Participants}

Enrolled children were divided into two groups according to their SH smoke exposure: group I (SHS children) and group II (controls or non SHS children).

\section{1-Group I (SH smoker children)}

It included 45 Egyptian children. They were 32 males (74.4\%) and

*Corresponding author: Eman Ahmed Zaky, Department of Pediatrics, Faculty of Medicine, Ain Shams University, Cairo, Egypt. E-mail: emanzaky@hotmail.com

Received March 21, 2014; Accepted May 10, 2015; Published May 17, 2015

Citation: Zaky EA, Fouda EM, Nabih ES, Youssef OI, Mohamed NH (2015) Association of Second Hand Smoking with Mental Disorders in Children- An Egyptian Study. J Psychol Abnorm Child 4: 140. doi:10.4172/2329-9525.1000140

Copyright: ( 2015 Zaky EA, et al. This is an open-access article distributed under the terms of the Creative Commons Attribution License, which permits unrestricted use, distribution, and reproduction in any medium, provided the original author and source are credited. 
13 females (25.6\%).Their ages ranged from 4 to 12 years with a mean age of $7.9 \pm 2.4$ years; all of them had at least one smoking parent and or were exposed to environmental tobacco smoke.

\section{2-Group II (controls/non SHS children)}

It included (30) children. They were 22 males (73.3\%) and 8 females (26.6\%). Their ages ranged from 4 to 12 years with a mean age of 7.6 \pm 2.5 years; all of them had neither history of parental smoking nor exposures to environmental tobacco smoke.

\section{Inclusion criteria:}

- $\quad$ Age ranged from 4 to 12 years

- Clinically physically healthy

- $\quad$ No sex predilection

\section{Exclusion criteria:}

Chronic physical illnesses and or handicaps which may affect psychometric scoring system.

Early active smoking

\section{Procedure}

1. Full medical history taking from enrolled caregivers (the mothers) for children younger than 10 years or from studied children who were older than 10 years laying stress on:

Personal history (Age, sex, residency especially that was near smoking areas)

\section{Exposure to smoking was explored as follows}

Household active smokers as fathers, mothers, brothers, or others, type of their smoking (cigarette or hookah), Number of cigarettes consumed by parents or others /day, was smoking indoor or outdoor?, Was there any area of smoking around the house (e.g., gas station, baking shops)?, was there exposure to smoking during antenatal period from fathers or mothers during first, second, or third trimesters?, Smoking index : number of cigarettes smoked per day $\times$ number of years smoked) $/ 20$, how many cigarettes did the active smoker member smoke on a typical day? ${ }^{*} 10$ or less, ${ }^{\star} 11-20,{ }^{*} 21-30,{ }^{\star} 31$ or more, did the active smoker member smoke in presence of the child: every day, rarely, or not?, was the active smoker member aware of the hazards of smoking on his or her children?, did the active smoker member smoke during examination to be enrolled in the study?, was the active smoker member still a smoker or an ex-smoker?

Was there any problem with child birth weight?

Did the child suffer from recurrent chest infections?

2- Thorough clinical examination with special emphasis on free cardiac and chest examination of the enrolled children.

\section{Questionnaires/ Instruments}

a. Psychosocial function assessment using an arabic validated version of Pediatric Symptom Checklist (PSC) Pediatric Symptom Checklist (PSC) [7]

The Pediatric Symptom Checklist $[8,9,10]$ is a psychosocial screen designed to facilitate the recognition of cognitive, emotional, and behavioral problems so that appropriate interventions can be initiated as early as possible. It consists of 35 items that are rated as "Never," "Sometimes," or "Often" present and scored as 0, 1, and 2, respectively. The total score was calculated by adding the score for each of the assessed 35 items:
- For children and children ages 6 -16, a cutoff score of 28 points from total 70 points or higher indicated psychological impairment.

- For children ages 4 - 5 years, the PSC cutoff score is 24 points or higher.

- Items that are left blank (empty) are simply ignored (i.e., score equals 0 ).

- If four or more items are left blank, the questionnaire was considered invalid

A positive total score on the PSC suggested the need for further evaluation by a qualified health or mental health professional as both false positives and false negatives occur. To determine what kinds of mental health problems were encountered among studied children, three factor scores on the PSC were considered:

\section{*The PSC Attention Subscale that consisted of 5 items:}

Children who received 5 or more points on it needed further assessment

\section{*The PSC Internalizing Subscale consisted of 5 items:}

It is a screen for anxiety and depression.Children who received 5 or more points on it needed further assessment

\section{*The PSC Externalizing Subscale consisted of 7 items:}

It is a screen for conduct disorder and oppositional defiant disorder and rage disorder. Children who received 7or more point needed further assessment

\section{b- Strengths and Difficulties Questionnaire (SDQ) [11-13]}

Psychological distress was assessed using an Arabic validated parental version of the Strengths and Difficulties Questionnaire (SDQ). SDQ consists of 25 items which has demonstrated good reliability and validity. The main outcome was total difficulties score (ranging from $0-40$ ), with a higher score representing worse mental health. The first 25 items in the SDQ comprise 5 subscales (emotional symptom scale, conduct problem scale, hyperactivity scale, peer problem scale, and prosocial scale) of 5 items each. It is usually easiest to score all scales before working out the Total Difficulties Score. For data entry, the responses to items should always be entered the same way, but they are not all scored the same way. For each of the scales the score can range from 0-10 if all 5 items were completed. Scale scores can be prorated if at least 3 items were completed.

Calculating the Summary scores for the five SDQ Subscales (Subscale Scores)

Standard values were used for coding Item responses and Summary scores. The standard values for coding individual Item responses were 0 (Not True), 1 (Somewhat True), 2 (Certainly True); and the missing values (Unable to rate). The exceptions were items $07,11,14,21$ and 25 . These items were "reverse-scored", that is, the standard value was mapped (0) to Item scores as follows) $0 \rightarrow 2,2 \rightarrow 0,1 \rightarrow 1$ ).

\section{Calculating the Total Difficulties scores}

The simplest way to calculate the total difficulties score was to add up the following summary scores with the result being rounded to the nearest whole number:

Total Score $=$ Emotional Scale + Conduct Scale + Hyperactivity Scale + Peer Problem Scale

c- DSM IV TR $(2000)$ [14,15] diagnostic criteria were used to settle the diagnosis of any suspected mental disorder. 


\section{d- Assessment of mental age}

Mental age assessment was done using an Arabic validated version of Standford Binet test, 5 th edition to calculate the intelligence quotient (IQ). This test was used to measure the child's cognitive abilities. It is suitable for children aging from 2-16 years. The test has 2 items, the verbal and the performance IQs and the test item was chosen according to the child's abilities. IQ was calculated by dividing the mental age by the chronological age multiplied by 100 . Subnormal intellectual function is diagnosed when IQ is below $70[16,17]$.

\section{Biological assessment of SHS}

Assessment of cotonine level in urine was done using the Cotinine (Urine) Microplate ELISA which is a competitive enzyme immunoassay for the detection of Cotinine in human urine. The wells of the microtitre strips were coated with anti-Cotinine antibody. During the first incubation, the horseradish peroxidase (HRP) labelled Cotinine competed with the free Cotinine in the donors sample for the antiCotinine antibody binding sites on the microtitre strips. The wells were washed to remove any excess enzyme material prior to the addition of the TMB substrate solution. Addition of the stop solution terminated the reaction and absorbances were read spectrophotometrically at 450 $\mathrm{nm}[18,19]$.

\section{Data analysis}

Analysis of the obtained data was done by IBM computer using SPSS (statistical program for social science version 16) [20] as follows: description of quantitative variables as means, SDs, and ranges, description of qualitative variables as numbers and percentages. Chi-square test was used to compare qualitative variables between groups. Unpaired t-test was used to compare quantitative variables, in parametric data ( $\mathrm{SD}<50 \%$ mean), Mann Whitney Willcoxon test was used instead of unpaired t-test in non parametric data ( $\mathrm{SD}>50 \%$ mean). Spearman Correlation co-efficient " $\mathrm{r}$ " test was used to rank variables versus each other positively or inversely. At the study sample, the calculated study power was 0.82 . Results were considered statistically insignificant at $p>0.05$, significant at $p<0.05$, and highly significant at $\mathrm{p}<0.01$. Receiver Operating Characteristic Curve (ROC) was plotted to define the sensitivity and specificity of PSC \& SQD in diagnosis of mental disorders in the studied sample.

\section{Results}

All the studied SHS children (group I) were exposed to their fathers cigarette smoke during both pre and postnatal periods. Twenty eight percent of their parents smoked 11-20 cigarettes per day. Sixty two percent of SHS children lived nearby smoking areas and $40 \%$ of them had history of recurrent chest infections.

SHS children had significantly higher mean value of PSC total score compared to controls indicating a significant degree of psychosocial dysfunction of those children $(\mathrm{p}<0.001)$. Also, the mean value of total SDQ scores was significantly higher in SHS children compared to controls $(\mathrm{p}<0.05)$ representing a significant psychological stress indicative of worse mental health. Mean values of ADHD, internalization behavior, and externalization behavior sub-scores of PSC were also significantly higher in SHS children compared to controls; $\mathrm{p}<0.05$, $\mathrm{p}<0.05$, and $\mathrm{p}<0.001$ respectively (Table 1 ).

Psychosocial dysfunction (PSD) as judged by the total PSC score was significantly more prevalent among SHS children $(93.3 \%)$ compared to controls $(53.3 \%) ; \mathrm{p}<0.001$. On the other hand, clinically significant combined disorders were frequently more encountered among SHS children (57.8\%) compared to controls (20\%); $\mathrm{p}<0.001$.

\begin{tabular}{|c|c|c|c|c|c|c|c|}
\hline \multirow[t]{2}{*}{ GroupVariables } & \multicolumn{2}{|c|}{$\begin{array}{l}\text { Group I SHS } \\
\text { children } \\
(\text { No }=45)\end{array}$} & \multicolumn{2}{|c|}{$\begin{array}{l}\text { Group II } \\
\text { Controls } \\
(\text { No }=30)\end{array}$} & \multirow[t]{2}{*}{$t / z$} & \multirow[t]{2}{*}{$\mathbf{P}$} & \multirow[t]{2}{*}{ Sig } \\
\hline & Mean & $\pm \mathrm{SD}$ & Mean & $\pm S D$ & & & \\
\hline Total PSCscore & 29.8 & \pm 2.6 & 20.7 & \pm 9.0 & 6.0 & $<0.001$ & HS \\
\hline TotalSDQ score & 15.2 & \pm 5.0 & 11.4 & \pm 5.0 & 2.8 & $<0.05$ & $S$ \\
\hline ADHD PSCsubscore & 5.1 & \pm 2.1 & 3.6 & \pm 1.5 & 2.6 & $<0.05$ & $S$ \\
\hline $\begin{array}{l}\text { Internalization behavior } \\
\text { PSCsubscore }\end{array}$ & 4.9 & \pm 2.0 & 3.5 & \pm 1.6 & 2.45 & $<0.05$ & $\mathrm{~S}$ \\
\hline $\begin{array}{l}\text { Externalizationbehavior } \\
\text { score }\end{array}$ & 9.0 & \pm 2.7 & 6.1 & \pm 3 & 4.0 & $<0.001$ & HS \\
\hline $\begin{array}{l}\text { Emotion SDQ sub } \\
\text { score }\end{array}$ & 4.3 & \pm 2.0 & 3.4 & \pm 2.5 & $1.78^{*}$ & $>0.05$ & NS \\
\hline $\begin{array}{l}\text { Conduct SDQsub } \\
\text { score }\end{array}$ & 3.7 & \pm 2.2 & 2.9 & \pm 2.1 & $1.6^{*}$ & $>0.05$ & NS \\
\hline ADHD SDQ subscore & 5.3 & \pm 2.0 & 3.5 & \pm 1.6 & $4.0^{*}$ & $<0.001$ & HS \\
\hline $\begin{array}{l}\text { Peer SDQ sub problem } \\
\text { score }\end{array}$ & 2.0 & \pm 2.1 & 1.7 & \pm 1.6 & $0.7^{*}$ & $>0.05$ & NS \\
\hline $\begin{array}{l}\text { Prosocialproblem SDQ } \\
\text { sub score }\end{array}$ & 6.0 & \pm 2.6 & 7.7 & \pm 1.8 & $3.0^{*}$ & $<0.001$ & HS \\
\hline IQ & 87.9 & \pm 6.0 & 91.3 & \pm 1.0 & $2.0^{*}$ & $<0.05$ & $\mathbf{s}$ \\
\hline $\begin{array}{l}\text { Cotinine(ng/mg } \\
\text { creatinine) }\end{array}$ & 77.3 & \pm 8.1 & 52.2 & \pm 7.5 & $3.0^{*}$ & $<0.001$ & HS \\
\hline
\end{tabular}

*Mann Whitney test " $z$ ", $t^{\star *}=$ unpaired t-test, PSC=pediatric Symptom checklist $S D Q=$ strength and difficulties questionnaire, $I Q=$ Intelligence Questionnaire $p>0.05=$ Statistically non significant, $P<0.05=$ statistically significant $p<0.01=$ statistically highly significant

Table 1: Statistical comparison between studied groups as regards the mean values of different studied quantitative variables.

Also, clinically significant ADHD and internalization subs-cores were more encountered among the former group compared to the later one but the comparison was only on descriptive basis as children number in these subgroups were small. Meanwhile, clinically significant externalization sub-score was less prevalent in SHS children (15.6\%) compared to controls $(26.7 \%) ; \mathrm{p}<0.001$.

Mean values of emotion, conduct, ADHD, and peer problem SDQ sub-scores were higher in SHS children compared to controls but the comparison was only statistically significant for ADHD sub-score. Meanwhile, prosocial problem sub-score was significantly lower in SHS children than that of controls; $\mathrm{p}<0.001$ (Table 1).

Interpretation of SDQ total scores revealed significantly higher frequency of "may have a disorder" (30.2\%), "suggested to have a disorder" (53.5\%), and "have combined disorders" (48.8\%) in SHS children compared to controls. Meanwhile, "have a single disorder" was also more prevalent among SHS children (28.8\%) compared to controls but the number of control children in that subgroup only permitted descriptive statistical analysis. Clinically significant behavioral or psychiatric disorders according to SDQ subscales scores revealed that they were all (conduct disorder, ADHD, emotional disorder, and prosocial and peer problems) more prevalent among SHS children compared to controls but the difference was statistically significant only concerning emotional problems $(\mathrm{p}<0.05)$.

Using SDQ subscales, the current study showed that $40 \%$ of SHS children had conduct disorder compared to $13.3 \%$ of control group and $20 \%$ of SHS children may have conduct disorder compared to $6.6 \%$ of controls. Also, 33.3\% of SHS children had ADHD disorder compared to $3.3 \%$ of control group and $11.1 \%$ of SHS children may have ADHD disorder compared to $3.3 \%$ of controls. Furthermore, $40 \%$ of SHS children had emotional problem compared to $20 \%$ of control group and 
$22.2 \%$ of SHS children may have emotional problem compared to $6.6 \%$ of controls. On the other hand, $13.3 \%$ of SHS children had peer problem compared to none of control group and $6.6 \%$ of SHS children may have peer problem compared to $3.3 \%$ of controls. Prosocial problem was recorded in $37.7 \%$ of SHS children compared to $3.3 \%$ of control group and $15.5 \%$ of SHS children was found to have "may have prosocial problem" interpretation compared to $33.3 \%$ of controls.

SHS children who were proven to have PSD according to PSC total score cut-off value were diagnosed as "may have a problem" in $31.1 \%$ and "have a problem" in $37.7 \%$ of them. Also, SHS children had significantly lower mean IQ $(87.9 \pm 6.0)$ compared to controls $(91.3 \pm$ $1.0) ; \mathrm{p}<0.05$ (Table 1 ).

Urinary cotinine mean value was significantly higher among SHS children $(77.3 \pm 8.1 \mathrm{ng} / \mathrm{mg}$ creatinine) compared to controls $(52.2 \pm$ $7.5 \mathrm{ng} / \mathrm{mg}$ creatinine); $\mathrm{p}<0.001$ (Table 1 ). The present study showed statistically insignificant difference in the mean urinary cotinine level in SHS females ( $85 \pm 7.3 \mathrm{ng} / \mathrm{mg}$ creatinine) compared to SHS males children $(87 \pm 9.8 \mathrm{ng} / \mathrm{mg}$ creatinine), $\mathrm{p}$ value $(>0.05)$. Statistically insignificant difference in the mean urinary cotinine level among studied SHS children who lived nearby smoking areas $(87 \pm 9.8 \mathrm{ng} / \mathrm{mg}$ creatinine) compared to those who lived far from smoking areas (85 \pm $7.3 \mathrm{ng} / \mathrm{mg}$ creatinine); $\mathrm{p}>0.05$. Mean values of total PSC and SDQ scores and urinary cotinine level did not differ significantly when studied SHS children who were living near smoking areas when compared with those who were not; $\mathrm{p}>0.05$ for all.

Regarding the interpretation of SDQ total scores of studied children who were living near smoking areas compared with those who were not, it showed that "may have a disorder" was recorded in 11 out of 29 lived near smoking area (37.9\%) compared to 5 out of 16 who did not live near a smoking are (31.2\%), "suggested to have a disorder" was recorded in 16 of the former subgroup (55.1\%) compared to 5 of the later group (31.2\%) while "do not have disorders" was recorded in 2 $(6 \%)$ of the former group compared to $6(37.5 \%)$ of the later group.

Mean values of total PSC and SDQ scores and their subscale scores and urinary cotinine level did not differ significantly when studied male SHS children compared with studied female SHS children; $p>0.05$ for all.

ADHD "may have a disorder" was encountered in $12.9 \%$ of studied male SHS children compared to $7.1 \%$ of studied female SHS children while ADHD "have a disorder" interpretation was recorded in $38.7 \%$ of studied male SHS children compared to $21.4 \%$ of studied female SHS children.

There were statistically insignificant correlations between smoking index, smoking degree, and urinary cotinine level with birth weight, current weight, height, and body mass index of studied SHS children; $\mathrm{p}>0.05$ for all. Also, there were statistically insignificant correlations between smoking index, smoking degree, and urinary cotinine level with IQ, PSC total score, PSC ADHD subscore, and PSC externalization and internalization behavior subscores of studied SHS children; $p>0.05$ for all except for the positive correlation that was recorded between the degree of smoking and internalization behavior subscore which was statistically significant; $p<0.05$. In studied SHS children, there were statistically significant positive correlations between ADHD subscore of SDQ and smoking index and between emotion subscore of SDQ and degree of smoking; $\mathrm{p}<0.05$ for both. Meanwhile, there was statistically significant negative correlation between prosocial subscore of SDQ and urinary cotinine level; $\mathrm{p}<0.05$ (Tables 2 and 3 ).

DSM IV TR diagnostic criteria were used to settle the diagnosis of

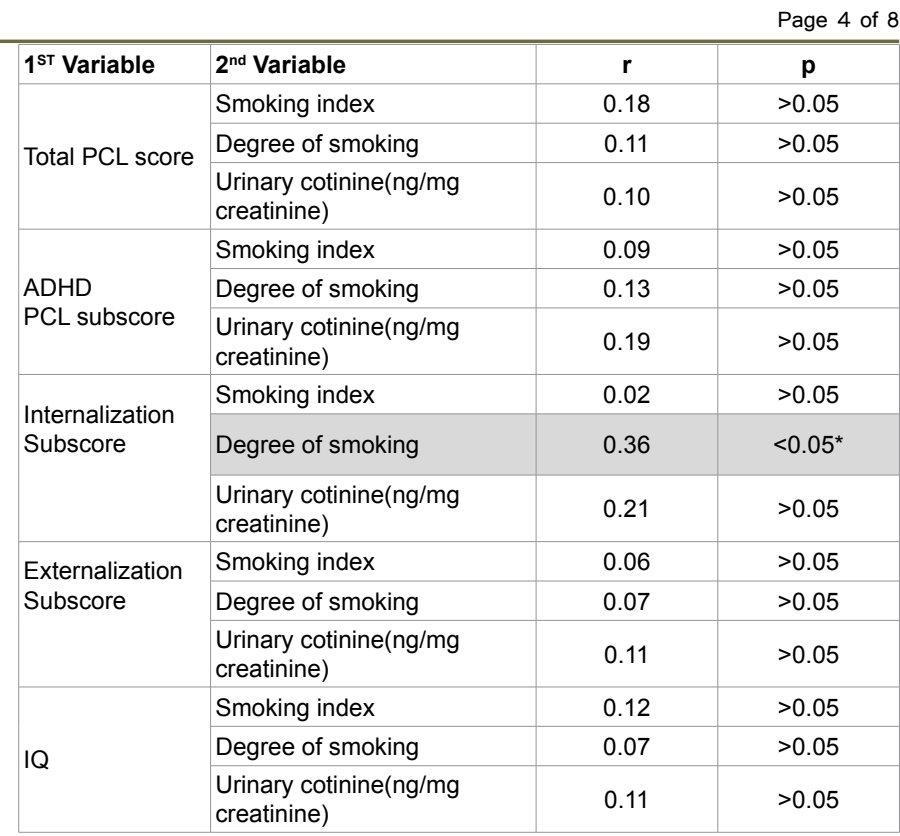

(Smoking index $=$ number of cigarettes smoked per day $\times$ number of years smoked/20)

(Degree of smoking = no of cigarette/ day)

$p>0.05=$ statistically insignificant, $p<0.05=$ statistically significant

$r=$ Spearman correlation test,$\quad I Q=$ intelligence quotient, $P S C=$ Pediatric Checklist ADHD=Attention Hyperactivity Disorder

Table 2: Correlations between smoking index, smoking degree, and urinary cotinine levels with IQ, PCL total score, and its ADHD, externalization behavior, and internalization behavior subscores in studied SHS children.

\begin{tabular}{|c|c|c|c|}
\hline $1^{\text {ST }}$ Variable & $2^{\text {nd }}$ Variable & $r$ & p \\
\hline \multirow{3}{*}{ Total SQD score } & Smoking index & 0.18 & $>0.05$ \\
\hline & Degree of smoking & 0.16 & $>0.05$ \\
\hline & $\begin{array}{l}\text { Urinary cotinine } \\
\text { (ng/mg creatinine) }\end{array}$ & 0.17 & $>0.05$ \\
\hline \multirow[t]{3}{*}{ Emotion subscore } & Smoking index & 0.20 & $>0.05$ \\
\hline & Degree of smoking & 0.48 & $<0.05$ \\
\hline & $\begin{array}{l}\text { Urinary cotinine } \\
\text { (ng/mg creatinine) }\end{array}$ & 0.18 & $>0.05$ \\
\hline \multirow{3}{*}{$\begin{array}{l}\text { Conduct } \\
\text { Subscore }\end{array}$} & Smoking index & 0.02 & $>0.05$ \\
\hline & Degree of smoking & 0.17 & $>0.05$ \\
\hline & $\begin{array}{l}\text { Urinary cotinine } \\
\text { (ng/mg creatinine) }\end{array}$ & 0.19 & $>0.05$ \\
\hline \multirow{3}{*}{$\begin{array}{l}\text { ADHD } \\
\text { Subscore }\end{array}$} & Smoking index & 0.45 & $<0.05$ \\
\hline & Degree of smoking & 0.04 & $>0.05$ \\
\hline & $\begin{array}{l}\text { Urinary cotinine } \\
\text { (ng/mg creatinine) }\end{array}$ & 0.13 & $>0.05$ \\
\hline \multirow{3}{*}{ Peer subscore } & Smoking index & 0.01 & $>0.05$ \\
\hline & Degree of smoking & 0.13 & $>0.05$ \\
\hline & $\begin{array}{l}\text { Urinary cotinine } \\
\text { (ng/mg creatinine) }\end{array}$ & 0.18 & $>0.05$ \\
\hline \multirow{3}{*}{ Prosocial subscore } & Smoking index & -0.04 & $>0.05$ \\
\hline & Degree of smoking & 0.02 & $>0.05$ \\
\hline & $\begin{array}{l}\text { Urinary cotinine } \\
\text { (ng/mg creatinine) }\end{array}$ & -0.55 & $<0.05$ \\
\hline
\end{tabular}

(Smoking index $=$ number of cigarettes smoked per day $\times$ number of years smoked/20)

(Degree of smoking = no of cigarette/ day), ADHD = Attention Deficit Hyperactivity Disorder

$p>0.05=$ statistically insignificant, $p<0.05=$ statistically significant

$\mathbf{r}=$ Spearman correlation test, $S D Q=$ Strength Difficulty Questionnaire

Table 3: Correlations between smoking index, smoking degree, and urinary cotinine levels with SDQ total score, and its subscores in studied SHS children. 


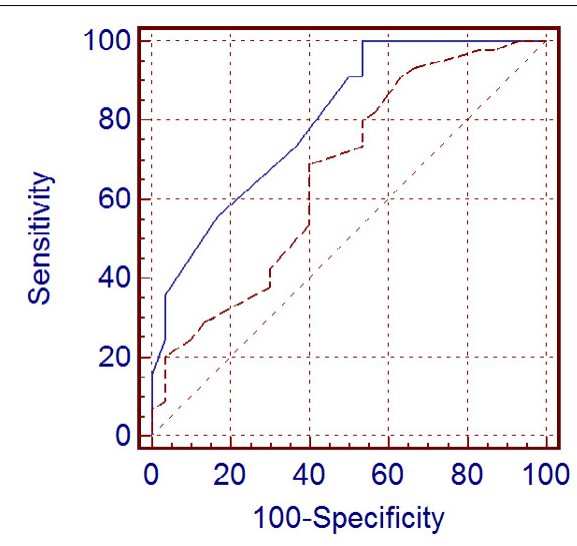

Figure 1: Receiver Operating Characteristic (ROC) curve defining for both SDQ and PSC total scores showing the statistical difference in sensitivity and specificity for both the total scores of PSC \& SDQ.

any suspected mental disorder in the studied children by using PSC \& SQD, then Receiver Operating Characteristic curve (ROC curve) was plotted to define the sensitivity and specificity of PSC \& SQD in accurate diagnosis of mental disorders in the studied sample. It showed that PSC total score had a sensitivity of $100 \%$, a specificity of $46.7 \%$, a PPV (positive predictive value) of $73.8 \%$, and a NPV (negative predictive value) of $100 \%$ with a diagnostic accuracy of $80 \%$. On the other hand, ROC curve showed that SDQ total score had a sensitivity of $68.9 \%$, a specificity of $60 \%$, a PPV (positive predictive value) of $72.1 \%$ and a NPV (negative predictive value) of $56.2 \%$ with a diagnostic accuracy of $66 \%$. Also, a statistically significant difference between SDQ and PSC total scores as regards sensitivity and specificity was documented (Figure 1). The difference between area under the curve between SDQ and PSC was 0.13 with a $\mathrm{p}$ value of $<0.05$.

\section{Discussion}

Previous cross-sectional and prospective studies suggested that exposure to SHS may precipitate the onset of or exacerbate symptoms of mental disorders [4,21]. So, the current cross sectional study aimed at investigating the potential association between SHS exposure and emotional, behavioral, and or psychiatric problems in a group of Egyptian children as to the authors' knowledge, such an association have not been studied before in our country.

All the studied SHS children (group I) were exposed to their fathers' cigarette smoke during both pre and postnatal periods. Twenty - eight percent of their parents smoked 11-20 cigarettes per day. Only 3 fathers smoked outdoor with their children rarely exposed to their smoke and 42 fathers smoked both indoor and outdoor with their children exposed everyday to their smoke. Sixty two percent of SHS children lived nearby smoking areas (baking shops, gas station and coffee shops).

Concerning psychometric assessment, two short screening tests have been used to evaluate the enrolled children in the present study, strength and difficulties questionnaire (SDQ) and Pediatric Symptom Check List (PSC). Reviewing the literature, no researches have used the PSC in evaluation of mental health among children exposed to SHS. So, the combination of SDQ \& PSC screening tools seemed worthy of investigation comparing them concerning their specificity and sensitivity in diagnosing behavioural and or psychiatric disorders in our studied sample.

Using SDQ, a statistically significant increase in the mean total SDQ score in SHS children $(15.2 \pm 5.0)$ compared to controls (11.4 \pm 5.0) was recorded; $p<0.05$ indicating increased risk to psychiatric problems in SHS children. This came in agreement with Seungmi et al. [22] who found that children exposed to pre and post natal maternal smoking or those exposed to postnatal paternal smoking had higher total SDQ score compared to non-exposed children. They attributed this to unmeasured genetic or environmental family factors combined with biological effects of tobacco exposure.

In our study using SDQ total score, $53.5 \%$ of SHS children had psychiatric problem compared to $33.3 \%$ of controls; $\mathrm{p}<0.05$ while $30.2 \%$ of SHS children "may have" psychiatric problem compared to 23.3\% of controls $(\mathrm{p}<0.05)$. This was in agreement with Simon et al. [23] who found that children exposed to pre and postnatal SHS had twice estimated risk of having abnormal total SDQ. They attributed this to neurotoxicity of tobacco toxic substances as well as its vasoconstrictor nature of nicotine leading to neurotoxic and hypoxic effects on the fetal-placental unit and on the developing nervous system.

As regards (SDQ) subscales results, the mean value of ADHD score in our sample of SHS children was significantly higher $(5.3 \pm 2.0)$ compared to controls $(3.5 \pm 1.6)$; $\mathrm{p}$ value $(<0.001)$ indicating a higher risk to ADHD in SHS children. This came in agreement with Hamer et al. [24] \& Seungmi et al. [22]. Neuman et al. [25] also suggested a possibility that needs to be explored about gene-environmental interactions mediating the relationship between SHS and ADHD.

On the other hand, a statistically significant lower value of the mean prosocial problem scores in our enrolled SHS children $(6.0 \pm$ 2.6) compared to controls $(7.7 \pm 1.8), \mathrm{p}<0.001$ indicating a significant increase of prosocial problems among SHS exposed children. This came in agreement with Hamer et al. [24] who also showed that the mean value of conduct problem score was higher in their studied SHS children compared to controls but the difference did not reach statistical significance. Similarly, Seungmi et al. [22] found that children exposed to maternal or parental smoke had higher conduct score.

In the current study, statistically insignificant differences were found between both studied groups as regards the mean values of emotional and peer problem SDQ subscores; $\mathrm{p}>0.05$ for both. This was in disagreement with Hamer et al. [24] who found an increase in the emotional score in SHS children while Simon et al. [23] after adjustment of unmeasured factors as socioeconomic standards and parent's psychological status found no increase of the emotion score in SHS children.

Using SDQ subscales, the current study showed that conduct disorder and emotional problems were the most prevalent disorders among studied SHS exposed children compared to controls followed by $\mathrm{ADHD}$, and peer problems respectively. Such findings were similar to those of Twardella et al. [26] who found that among SHS children, the conduct problems was the most frequently observed and hyperactivity/ inattention was their least frequently observed. Deficiencies in prosocial behaviors were also found in their sample.

In the present study, the mean value of total PSC score was found to be significantly higher in SHS children compared to controls; $p<0.001$ indicating psychosocial dysfunction susceptibility together with increased risk of psychiatric problems in SHS children. Hook et al. [27] and Robinson et al. [28] used child behavior check list (CBCL) to assess the children exposed to smoking mothers during and after pregnancy and attributed the increase in risk to psychiatric problems in those children to direct effect of nicotine on the developing brain.

Child Behavior Checklist (CBCL) composed of 120 items which does not meet the practical requirements in the limited time available 
for a standard health examination and difficult in calculation of its total score while PSC which was used in the current study composed of 35 items and it is easy to calculate its total score. Using PSC, 93.3\% of studied SHS children were suggested to have psychiatric problems compared to $53.3 \%$ of Controls; $(\mathrm{p}<0.001)$.

Poole et al. [5] used another type of psychometric assessment which was Columbia Impairment Scale (CIS) and also demonstrated increased rates of SHS children's behavioral and emotional problems and suggested a combination of the direct effect of SHS exposure on the developing brain with other biological and psychosocial factors ultimately shaping child behavior.

Using PSC, this study revealed significantly higher mean values of ADHD, externalization behavior, and internalization behavior sub-scores among SHS children compared to controls indicating an increased risk of those disorders in such children. Similarly, Brion et al. [29] found an association between parental smoking and greater offspring hyperactivity, externalization behavior problem, and internalization problems but by using child behavior checklist (CBCL).

As regards subscales of PSC scores , 11.1\% of SHS children had only internalization behavior problem, $8.9 \%$ had only ADHD , and $57.8 \%$ had combined disorders while $15.6 \%$ of those children had only externalization behavior problem. Using CBCL in assessing SHS children, Rodriguez et al. [30] found only ADHD problems while Hook et al., [27]found only externalizing problem.

In the current study, mean value of IQ in tested SHS children was significantly lower compared to tested controls; $p$ value $<0.05$.This is in agreement with Seungmi et al. [22] who explained that by combination of direct effect of smoking with parental factors as parental education and parental IQ or environmental factors as socioeconomic standard on the developing brain.

Concerning the concentration of urinary cotinine, it was considered by the Surgeon General Report [31] to have several advantages over blood or saliva assays such as the availability of large volume that usually can be collected, easily obtainable, and its level average is usually 5-6 times higher than serum levels for unconjugated cotinine. So, urinary cotinine has been chosen in the current study as a biomarker of passive smoking and it has been proved to be significantly higher among second hand smokers when compared with controls. The levels of urinary cotinine reflect more accurately the amount of environmental tobacoo smoke (ETS) exposure [32]. The foregoing result of the current study came in agreement with Thaqi et al. [33] and Puig et al. [34] who found that the urinary cotinine level is 1.4 times higher in children of smoking father and 3.5 times higher in children of smoking mother compared to non smoking households.

In the present study, statistical insignificant differences were found between SHS females and males studied children as regards the mean value of total PSC and SDQ scores and their different subscales. Hook et al. [27] found that both sexes were equally affected using CBCL. However, Hamer et al. [24] found that girls had lower SDQ scores compared to boys of the same age after adjustment of the surrounding socioeconomic factors and parental psychology of both sexes.

Using SDQ, this study revealed that $38.7 \%$ of SHS males had ADHD compared to $21.4 \%$ of SHS females. Twardella et al. [26] and Bandiera et al. [4] found significant difference between males and females as regards ADHD, conduct problems, and peer relationships, prosocial problems and total SDQ score. They explained that by variations in cotinine metabolism or by other biological, social, or environmental factors that vary across sex and race/ethnicity.
In the target area served by Children's Hospital, Ain Shams University, families belonging to that target area are usually more concerned, as a socio-cultural habit, about physical and mental health of their boys than their girls. A fact that explains the excess male to female ratio that was recorded in our both studied groups but within SHS exposed children, statistically insignificant difference in the mean urinary cotinine level was found comparing males to females, $\mathrm{p}$ value $(>0.05)$. This finding was in agreement with Salmela-Aro et al. [35] who reported that sex has no influence on cotinine level. In contrast, Benowitz et al. + found that male sex and black race were associated with slower nicotine metabolism but they conducted their study on adults and not on children as in our study as at a younger age, sex hormones still don't play an important role in cotinine metabolism.

As for residency near smoking area, $55.1 \%$ of our studied SHS exposed children who lived nearby smoking areas had psychiatric disorders by using SDQ compared to $31.2 \%$ of those who lived far from smoking areas, $(\mathrm{p}<0.05)$. This may be explained by the toxic effect of carbon monoxide and benzene which found in both cigarette and biofuel smoke on the developing brain. Zorina [37] stated that carbon monoxide have complex adverse effects on the central nervous system, combined with damaging effects on the respiratory, cardio-vascular, and urogenital systems, and blood forming organs as well as the immune mechanism.

Statistically insignificant difference in the mean urinary cotinine level among studied SHS children who lived nearby smoking areas compared to those who lived far from smoking areas; $p>0.05$ that was in agreement with Hmwe et al. [38] who explained it by the simple fact that nicotine is found in cigarettes but not in biofuels.

The current study revealed a statistically significant positive correlation between the degree of smoking and emotion sub score of SDQ /internalization score of PSC indicating that the risk of emotional problem among children increased with increase in the number of cigarette smoked by their parents per day. Simon et al. [23] found a dose -response relationship between degree of smoking and the risk to emotional problems, ADHD, conduct, peer, and prosocial problems. In contrast Twardella et al. [26] found that the increase in exposure to cigarette smoke was associated with increased risk to ADHD and conduct disorder only and explained this by the effect of exposure to side stream smoke in utero or postnatally on brain cellular density and cell size.

A statistically significant negative correlation was found between urinary cotinine value and prosocial score in SDQ indicating increased prosocial problem symptoms with increased urinary cotinine level in the current study. Also, weak positive correlations between urinary cotinine and total SDQ or PSC scores and different SDQ and PSC subscale scores were recorded. This is in disagreement with Cho et al. [39] who found statistically positive correlation between urine cotinine levels and parental reports of symptoms of ADHD and explained this also by the biological effect of cotonine on the developing brain in the childhood period.

Other researchers used salivary or serum cotonine level in their studies. Hamer, et al. [24] found that the highest salivary cotinine level had significantly higher total SDQ scores compared with those in the lowest cotonine level $(\mathrm{P}=0.001)$ and their strongest associations with cotinine levels emerged for hyperactivity and conduct disorder. Also, Bandiera [4] found that serum cotinine level was most strongly associated with ADHD symptoms while there was a slightly significant association of serum cotinine level with symptoms of emotional and conduct disorders. 
The current study demonstrated also a statistically significant positive correlation between smoking index and ADHD score, $p<0.05$ indicating that prolonged and higher exposure to smoking is associated with more ADHD symptoms both quantitatively and qualitatively. This finding is in agreement with Simon et al. [23] who stated that continuous patterns of higher nicotine levels alter nor adrenaline and dopamine response and the current physiological hypothesis on ADHD is a relative shortage of nor adrenaline and dopamine.

Using ROC curve a statistically significant difference between SDQ and PSC total scores concerning their sensitivity and specificity in detection of behavioral and or psychiatric problems was elicited, $\mathrm{p}<0.05$ as PSC total score had a sensitivity of $100 \%$ and a specificity of $46.7 \%$ while SDQ total score had a sensitivity of $68.9 \%$ and a specificity of $60 \%$. Vogels et al. [40] found a comparable sensitivity and specificity between PSC, SDQ, and CBCL in detection of psychosocial dysfunction among primary school children in general.

In conclusion, a significant association between secondhand smoke (SHS) exposure and emotional, behavioral, and or psychiatric problems in the studied sample of Egyptian children was proved. Also, the combination of SDQ \& PSC screening tools seemed worthy as together they detected the behavioal and or child psychiatric disorders among studied children more accurately improving their individual diagnostic specificity and sensitivity.

\section{Recommendations}

From the current study, the authors recommend taking firm measures to decrease SHS exposure including prohibiting all forms of tobacco smoke promotion together with raising public awareness about the potential hazards of SHS especially its impact on mental health of children. Also, it seems wise to use psychometric screening for SHS exposed children aiming at early detection and prompt management of any behavioral or psychiatric disorders among them. Lastly but by no means least, further Egyptian studies on a nationwide scale are needed to define the actual magnitude of the problem in our country as well as the utilization of basic neuro-scientific techniques to explore the pathogenesis of SHS exposure impact on the normal neurodevelopment and its link to future occurrence of childhood mental disorders.

\section{References}

1. Yanyan L, Peter D (2012) Effects of secondhand smoke on airway secretion and mucociliary clearance. Front Physiol 3: 342

2. DiFranza JR, Aligne CA, Weitzman M (2004) Prenatal and postnatal environmental tobacco smoke exposure and children's health. Pediatrics 113:1007-15

3. Pershagen G (2011) Accumulating evidence on health hazards of passive smoking. Acta pardiatr $88: 490-492$

4. Bandiera FC, Richardson AK, Lee DJ, He JP, Merikangas KR (2011) Secondhand smoke exposure and mental health among children and aldolescents. Arch Pediatr Adolesc 165 :332-338

5. Poole ED, Ying-Hua L, Samantha B,Michael W (2010) Adult household smoking is associated with increased child emotional and behavioral . Environ Health Perspect 118:150-154

6. Helsinki (1998) The WMA declaration of Helsinki 1960 with recommendations on biomedical research on human subjects. Chirurgia (Bucur) 93 :138- 140

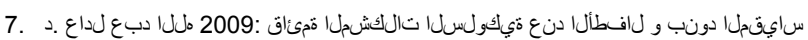

8. Little M, Murphy JM, and Jellinek MS (1994) Screening 4- and 5-year-old children for psychosocial dysfunction: Apreliminary study with the pediatric symptom checklist. Journal of Developmental and Behavioral Pediatrics 15: 191-197

9. Pagano M, Murphy JM, Pedersen M (1996) Screening for psychosocial problems in 4-5 year olds during routine EPSDT examinations: Validity and reliability in a Mexican-American sample. Clinical Pediatrics $35: 139-146$
10. Robinson J, Jellinek MS, Murphy JM (1988) Pediatric Symptom Checklist: Screening school-age children for psychosocial dysfunction. Journal of Pediatrics $112: 201-209$

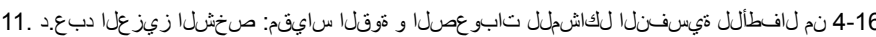
منس

12. Goodman R (1997) The Strengths and Difficulties Questionnaire: a research note. J Child Psychol Psychiatry 38:581-586

13. Goodman R, Renfrew D, Mullick M (2000) Predicting type of psychiatric disorder from Strengths and Difficulties Questionnaire (SDQ) scores in child mental health clinics in London and Dhaka. Eur Child Adolesc Psychiatry 13:129-134

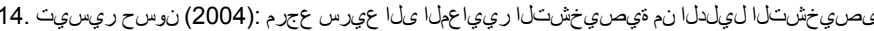

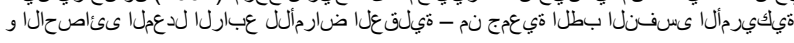

15. DSM IV TR (2000) American Psychiatric Association: Diagnostic and Statistical Manual of Mental Disorders, 4th Edition, Text GH Revision. Washington, DC, American Psychiatric Association

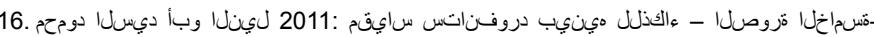

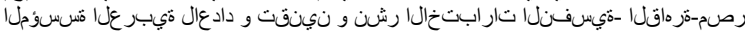

17. Bain S K, Allin J D (2005) Book review:Stanford-Binet intelligence scales, fifth edition. Journal of Psychoeducational Assessment 23: 87-95.

18. Benowitz NL (1996) Cotinine as a biomarker of environmental tobacco smoke exposure .Epidemiologic Review 18:188-204

19. Jung S, Lee IS, Kim SB, Moon CS, Jung JY, et al., (2012) Urine Cotinine fo Assessing Tobacco Smoke Exposure in Korean: Analysis of the Korea National Health and Nutrition Examination Survey (KNHANES). Tuberc Respir Dis $73: 210-8$

20. Statistical package for social science (2007) SPSS, program version 16. Spss Inc. SPSS for windows, version 16, Chicago, SpsS Inc

21. Herrmann M, Katherine K, Michael W (2008) Prenatal tobacco smoke and postnatal secondhand smoke exposure and child neurodevelopment. Current Opinion in Pediatrics 20: 184-190

22. Seungmi Y, Adriana, Michael S (2013) Exposure to parental smoking and child growth and development: a cohort study. BMC Pediatr 13: 104

23. Simon R, Peter R, Chih -Mei C (2010) prenatal and postnatal tobacco exposure and behavioral problems in 10-Year-Old Children: Results from the GINI-plus prospective birth cohort study. Environ Res 118 : 150-154

24. Hamer M, TamsinF, EmmanuelS, Samantha D, David B (2011) Objectively measured secondhand smoke exposure and mental health in children. Evidence From the Scottish Health Survey. Arch Pediatr Adolesc Med 165 326-331

25. Neuman RJ, Lobos E, Reich W, (2007) Prenatal smoking exposure and dopaminergic genotypes interact to cause a severe ADHD subtype. Biol Psychiatry 61:1320-1328

26. Twardella D, Bolte G, Fromme H, Wildner M ,von R (2010) Exposure to secondhand tobacco smoke and child behaviour - results from a cross-sectional study among preschool children in Bavaria. Acta Pædiatrica 99:106-111

27. Höök B, Cederblad M and Berg R (2006) Prenatal and postnatal maternal smoking as risk factors for preschool children's mental health. Acta Paediatr 95:671-7

28. Robinson M, Lean NJ, Oddy WH, Mattes E, Bulsara M, et al, (2010) Smoking cessation in pregnancy and the risk of child behavioural problems: a longitudinal prospective cohort study. J Epidemiol Community Health 64:622-9

29. Brion J, Cesar V, Alicia M, Bernardo HI, Colin S, et al (2010) Maternal smoking and child psychological problems: Disentangling causal and non-causal effects. Arch Gen Psychiatry 67: 841-9

30. Rodríguez ML, López VJ, Garrido R, Sacristán MA, Martínez RM, et al. S(2009) Translated, Estudio psicométrico-clínico de prevalencia y comorbilidad de trastorno por déficit de atención con hiperactividad en Castilla y León (España). Rev Pediatr Aten Primaria 11: 251-270

31. Surgeon General Report (2006) Health consequences of involuntary exposure to tobacco smoke: Department of Health and Human Services, Centers for Disease Control and Prevention, National Center for Chronic Disease Prevention and Health Promotion, Office on Smoking and Health. (www. surgeongeneral.gov/library/reports)

32. Fahmy AA (2010) Study of cord serum cotinine as a biomarker of fetal exposure 
Citation: Zaky EA, Fouda EM, Nabih ES, Youssef OI, Mohamed NH (2015) Association of Second Hand Smoking with Mental Disorders in ChildrenAn Egyptian Study. J Psychol Abnorm Child 4: 140. doi:10.4172/2329-9525.1000140

to nicotine by active and passive maternal smoking. Thesis submitted for partial fulfillment of in MD Pediatrics Faculty of Medicine, Ain Sham University under supervision Prof. Dr/ Osama NS

33. Thaqi A, Franke K, Merkel G, Wichmann HE, Heinrich J (2005) Biomarkers of exposure to passive smoking of school children: frequency and determinants. Indoor Air 15: 302-310

34. Puig C, Oscar GA, Toni M (2008) A longitudinal study of environmental tobacco smoke exposure in children: Parental selfreports versus age dependent biomarkers. BMC Public Health 8: 47

35. Salmela-Aro K, Tynkkynen L (2012) Gendered pathway in school burnout among adolescents. J Adolesc 35 : 929-939

36. Benowitz N, Johnstone E, Cargill A, Jacob R, Hinks L, et al (2009) Determinants of the rate of nicotine metabolism and effects on smoking behavior. Clin Pharmacol Ther 80: 319-330
37. Zorina IG (2012) Prenosological neuro-psychiatric disorders among schoolchildren and their relationship to environmental factors. Vestn Ross Akad Med Nauk : 30-4

38. Hmwe H, Katholiki $G$ and Michael H (2009) Maternal smoking, biofuel smoke exposure and child height-for-age in seven developing countries. Int. J. Epidemiol 38 : 1342-1350

39. Choa SC, Honga YC, Kim JW (2013) Association between urine cotinine levels, continuous performance test variables, and attention deficit hyperactivity disorder and learning disability symptoms in school-aged children Psychological Medicine 43 : 209-219

40. Vogels AG, Crone MR, Hoekstra F, Reijneveld SA (2009) Comparing three short questionnaires to detect psychosocial dysfunction among primary schoo children: a randomized method. BMC Public Health 9:489 